\title{
RETRACTION
}

\section{A Regulatory Circuit Mediating Convergence between Nurr1 Transcriptional Regulation and Wnt Signaling}

Hirochika Kitagawa, William J. Ray, Helmut Glantschnig, Pascale V. Nantermet, Yuanjiang Yu, Chih-Tai Leu, Shun-ichi Harada, Shigeaki Kato, Leonard P. Freedman

Department of Molecular Endocrinology, Merck Research Laboratories, West Point, Pennsylvania, USA; Institute of Molecular and Cellular Biosciences, University of Tokyo, Bunkyo-ku, Tokyo, Japan; ERATO, Japan Science and Technology Agency, Kawaguchi, Saitama, Japan

Volume 27, no. 21, p. 7486-7496, 2007. The publisher hereby retracts this article. After publication, this article was found to have multiple images that were unacceptably manipulated (Fig. 4B and 5A), a clear violation of ASM's ethical standards. Since the integrity of the data as presented was compromised, this publication is retracted in its entirety. We apologize to the readers of Molecular and Cellular Biology and regret any inconvenience this causes. 